\title{
Aportes teóricos derivados de las investigaciones sobre el lenguaje entre el 2000 y el 2010: una revisión*
}

\section{Theoretical Contributions Derived from Language Research Between 2000 and 2010: a Review}

\author{
J ohny Villada Zapata* \\ Universidad de Antioquia, Colombia \\ Liliana Chaves Castaño \\ Universidad de Antioquia, Colombia \\ Recibido: 16 de agosto de 2011 \\ Revisado: 4 de diciembre de 2011 \\ Aceptado: 20 de febrero de 2012
}

\section{Resumen}

El artículo presenta el análisis de contenido derivado de una revisión sistemática en la que se retomaron 57 estudios que tenían el lenguaje como tema central. Las investigaciones consultadas arrojan diversos aportes teóricos de los cuales sobresale, en primer lugar, el origen social de este proceso, tanto en la filogénesis como en la ontogénesis. En segundo lugar puede afirmarse que su desarrollo se ve afectado negativa o positivamente por variables como la nutrición, la relación con los padres, la socialización en la escuela y el ambiente social. Finalmente, la investigación con bebés muestra su participación activa en el reconocimiento del idioma, el acto de señalar como un hito importante en el desarrollo del lenguaje al provocar la atención conjunta y, finalmente, la relación existente entre el lenguaje y teoría de la mente.

Palabras claves: Lenguaje, investigaciones, aportes teóricos.

* $\quad$ Artículo de investigación.

** Correspondencia: Johny Villada Zapata, Universidad de Antioquia. Programa de Psicología Regionalizado. Correo electrónico: johnyvillada@ gmail.com. Dirección postal: Calle 67 № 53-108. Bloque 9 oficina 446. Medellín, Colombia. Liliana Chaves Castaño. Universidad de Antioquia, Colombia. Correo electrónico: lilianachaves1@gmail.com 


\section{Abstract}

This article presents an analysis of content derived from a systematic review which picked up 57 studies that had the language as a central theme. The consulted investigations cast various theoretical contributions from which protrudes, firstly, the social origin of this process in both the filogenesis and the ontogenesis. In second place it can be affirmed that their development is affected negative or positively by variables such as nutrition, relationship with the parents, socialization at school and social atmosphere. Finally, the research about babies shows their active participation in the recognition of language, the act of pointing out as an important landmark in language development when provoking the combined attention, and finally the existent relationship between language and theory of mind.

Keywords: Language, research, theoretical contributions.

\section{Introducción}

El lenguaje como tema importante para la psicología y las demás ciencias sociales ha gozado de una larga tradición teórica e investigativa que se ha preocupado por entender su funcionamiento, sus elementos constitutivos, su historia en la filogénesis de la especie y su expresión en el desarrollo individual.

Es por ello que vale la pena hacer constantes revisiones a la producción investigativa y teórica sobre este proceso psicológico, de modo que se puedan sintetizar los aportes realizados en un periodo de tiempo concreto, proporcionando una visión panorámica de las principales contribuciones en el campo.

La investigación presenta de forma sintética, diversos aportes teóricos derivados de la investigación en lenguaje durante la primera década del siglo XXI, en tópicos como la filogénesis, la ontogénesis, su contexto de desarrollo y la asociación con otros procesos psicológicos.

\section{Método}

El artículo presenta los hallazgos de una investigación de tipo revisión sistemática en la que se consultaron 57 estudios que tenían como tema central el lenguaje, publicados entre 2000 y 2010 en las bases de datos Ebsco - Psychology \& Behavioral Sciences Collection, Proceedings of the National Academy of Sciences of the United States of America PNAS, Red de Revistas Científicas de América Latina y el Caribe, España y Portugal Redalyc, Dialnet y Science Direct.

Para realizar dicha revisión sistemática se hicieron búsquedas hasta el 30 de diciembre de 2010 para el posterior proceso de selección de los artículos y su respectivo fichaje y análisis. El perfil de búsqueda usado fueron las siguientes palabras clave: [language], [language development] [language AND Brain]. Estas mismas claves de búsqueda se usaron en español.

Los criterios de inclusión fueron: publicaciones en revistas indexadas entre los años 2000 y 2010, artículos provenientes de las ciencias cognitivas 0 discursos afines, lenguaje como tema central de los estudios.

Los criterios de exclusión fueron: publicaciones en revistas no indexadas con fechas anteriores al año 2000, boletines, reseñas de libros, comentarios o perspectivas que no se deriven de investigaciones.

A continuación se presentan sintéticamente los principales aportes teóricos derivados de las investigaciones consultadas. 


\section{Aportes teóricos derivados de las investigaciones sobre el lenguaje}

Para Montealegre (2005) la actividad humana no versa solo en la dimensión de la acción práctica sobre los objetos, sino que cuenta con una serie de procesos que median entre ella y el mundo (siendo el lenguaje el principal de ellos). Es por ello que este proceso psicológico merece especial atención y la discusión de los hallazgos encontrados en la presente revisión sistemática retomará los aspectos centrales que aportaron a su teorización en la primera década del siglo XXI.

Para realizar este ejercicio vale la pena empezar por el tema de la filogénesis del lenguaje. Al respecto Steven Pinker (2010) apoyado en las ciencias cognitivas y la psicología evolutiva, propone que los homo sapiens evolucionaron al especializarse no solo en el nicho ecológico, sino en el nicho cognitivo definido como el ambiente mental ocupado por el razonamiento sobre la estructura causal del mundo, el problema de la cooperación con otras personas, el compartir el conocimiento y negociación de los acuerdos a través del lenguaje. Es por ello que este último coevolucionó con la inteligencia general (no evolucionaron por separado) permitiendo el éxito reproductivo, una sexualidad compleja, una mejora en la inversión parental por parte de ambos sexos, pero sobre todo, una adaptación a un grupo social (en el cual nace el lenguaje) y una acumulación de conocimientos locales y convenciones sociales.

En línea con el planteamiento que afirma que el lenguaje nace en los grupos sociales, los experimentos realizados por Kirby, Cornish \& Smith (2008) proporcionan evidencia empírica sobre la aparición del lenguaje como un logro social y no como la proeza de algún individuo. A un grupo de estudiantes universitarios sin experiencia en lingüística se les pidió que reprodujeran y enseñaran a otros un "idioma" (constituido por signos y sonidos arbitrarios sin ningún sentido, ni siquiera para los investigadores) de modo que pudieran empezar a comunicarse en dicho "idioma" y no en la lengua materna. De manera sorprendente, los participantes del estudio después de múlti- ples intentos y ensayos fueron solucionando los problemas de comunicación que tenían al principio y empezaron a transmitir mensajes que eran entendidos por los demás participantes de la investigación.

Los resultados anteriores tienen una estrecha relación con los modelos computacionales del lenguaje (Perruchet, 2005; J unyet 2001; Waibel 2001) que lo postulan como una gran adaptación lograda por los grupos sociales en su necesidad de transmitir un mensaje de una manera comprensible, en ausencia de un diseñador específico y dirigido para resolver problemas que van apareciendo en el nicho cognitivo. De modo que la hipótesis que se desprende es que así como en el laboratorio, en algún momento un grupo de individuos de la especie homo sapiens necesitaban transmitirse una serie de mensajes, pero solo poseían unos movimientos corporales (Spinney, 2003; De Vega, 2005) y unos sonidos arbitrarios producidos por su aparato fonador. Pero que como logro grupal pudieron coordinar dichos movimientos y sonidos para producir y reproducir mensajes dando lugar a las primeras formas del lenguaje.

Otra investigación que va en la línea de la evolución del lenguaje es la realizada por GoldinMeadow, Chee So, Zyurek \& Mylander (2008), quienes escogieron a cuarenta adultos hablantes nativos de cuatro idiomas distintos (Inglés, Turco, Español y Chino) y les pidieron que resolvieran una serie de tareas no verbales. De forma llamativa los hablantes de los cuatro idiomas utilizaron el mismo orden de movimientos en tareas no verbales. El orden utilizado fue el de: actor-objeto-acto, resultando este análogo al modelo sujeto-objeto-verbo que se encuentran en muchas lenguas del planeta. Lo anterior lleva a pensar a los investigadores que el lenguaje tuvo un origen común y que a pesar de la diseminación de lenguas en la geografía, todavía se conservan ciertas estructuras organizativas de las acciones, derivadas de esos inicios lingüísticos.

Ahora, si bien la adaptación al grupo social es aparentemente el principal motor de la aparición del lenguaje, no se puede olvidar que son necesarias una serie de estructuras anatómicas 
cerebrales que permitieran darle un piso biológico a lo que estaba sucediendo en el grupo social (Castaño, 2003; Fajardo, 2008; Sullivan \& Riccio, 2008). Es por ello que cobra especial importancia el meta-análisis realizado por Vigneau, Beaucousin, Hervea \& Duffauc (2006) en el que se retomaron estudios de neuroimagen que permitían conocer con mayor detalle las estructuras cerebrales relacionadas con distintos aspectos del lenguaje. A continuación se presentan brevemente los resultados de dicho meta-análisis.

Los informes de investigación consultados se clasificaron en dos componentes del lenguaje: 45 de ellos estaban relacionados con la dimensión fonológica del lenguaje y contaban con experimentos en los que los participantes debían repetir o articular sílabas, leer, escuchar 0 aparear a las sílabas o símbolos, leer una pseudo-palabra (construida sobre la ortografía de una lengua dada, pero sin sentido) contar el número de sílabas en una palabra o discriminar si una palabra termina con el mismo sonido. Estos estudios muestran una activación mayor en el lóbulo frontal izquierdo, seguido por la activación del lóbulo temporal izquierdo y una activación menor del lóbulo parietal también izquierdo.

De los estudios restantes 67 estaban dirigidos al procesamiento semántico del lenguaje e incluían experimentos en los que las personas debían acceder al significado de las palabras (leyéndolas o escuchándolas); realizando ejercicios de categorización (decidiendo si un estímulo presentado pertenece o no a los seres vivos); generando asociaciones (generando un verbo semánticamente relacionado con una palabra presentada); haciendo evocaciones semánticas (como la evocación de una propiedad del objeto), entre otras. Dichas investigaciones convergen en afirmar que en el procesamiento semántico hay una mayor activación del lóbulo temporal izquierdo, seguido por activaciones parietales también izquierdas.

Estos resultados proporcionan evidencia empírica de la hipótesis que afirma que hay una asociación entre el lóbulo frontal izquierdo y temporal izquierdo (con una menor participación del parietal) para la fonología y la semántica, contra- diciendo la disociación tradicional entre ambos procesos. De esta manera se asume la existencia de regiones cruce, donde se superponen áreas funcionales para el procesamiento fonológico y semántico y no solo una co-localización de estas áreas funcionales.

De lo expuesto hasta el momento se puede afirmar entonces que el lenguaje surgió como una adaptación de los grupos sociales y que su apoyo anatómico (fonológico y semántico) se encuentra en las zonas frontales y temporales del hemisferio izquierdo. Si lo anterior está claro, cabe la pregunta sobre ¿cómo se desarrolla el lenguaje en un individuo concreto en los primeros años de vida?, por lo cual vale la pena detenerse en los hallazgos producidos en torno a la ontogénesis del lenguaje.

En primer lugar vale la pena mencionar que hay una serie de estudios en el siglo XXI que se han detenido en las etapas o sucesos más tempranos en el desarrollo del lenguaje, teniendo a los bebés como objetos de investigación (Peña-Garay, 2005; Pettito, Holowka \& Sergio, 2001; Scerif, 2006). A continuación se presentan los resultados más importantes en esta línea:

Para Kuhl (2000) hay seis principios básicos evidentes en el lenguaje de los bebés que contradicen los clásicos postulados de tabula rasa de Skinner y la supremacía del aprendizaje en la adquisición del lenguaje, y de la gramática universal de Chomsky en una propuesta netamente innatista: (i) los bebés inicialmente analizan las unidades básicas de expresión de su lengua, lo que les permite adquirir unidades de orden superior creadas por sus combinaciones, (ii) el proceso de desarrollo no es seleccionista en tanto que las habilidades innatamente especificadas no seleccionan la experiencia que le corresponde en la vida de los bebés, (iii) en vez de ello, hay proceso de aprendizaje perceptivo que comienza con la exposición al lenguaje, durante el cual los bebés deben detectar patrones y explorar propiedades del mismo, (iv) hay un fuerte enlace entre la imitación, el acto de señalar, la percepción del habla y la producción temprana del lenguaje por parte de ellos, (v) los adultos frente a los niños 
inconscientemente modifican su discurso para que coincida con las estrategias que los bebés están construyendo, lo que resulta fundamental en una cartografía inicial de las palabras y (vi) el período crítico para el lenguaje se ve influenciado, no solo por tiempo sino por el compromiso con la experiencia de intercambios lingüísticos. Según estos principios no es posible pensar el desarrollo del lenguaje en términos de un aprendizaje asociativo exclusivo (Skinner), o de una capacidad innata para el lenguaje que requiere un poco estimulación ambiental (Chomsky).

Alguno de estos principios de los que habla Kuhl (2000) se ven apoyados por otras investigaciones que aportan evidencia a su favor. En primer lugar tenemos la realizada por Kinzler, Dupoux \& Spelke (2007) en la que se ubicó frente a un grupo de bebés (de 18 meses aprox.) unas imágenes de mujeres a quienes se les colocaba un audio en un idioma extranjero y después otras imágenes con su idioma nativo y así saber cuáles imágenes prefería el bebé. Como resultado se encontró que los niños tienden a preferir los rostros de las personas que hablan su mismo idioma, mientras que tienden a ignorar los rostros de las personas que hablan otros. Lo que es congruente con el principio (i) pues hay un reconocimiento aparente del idioma materno, dado por una exposición y percepción del mismo durante 18 meses.

Otra investigación que refuerza el enunciado anterior es la de Dehaene, Artiges \& Naccache (2007) en la que usaron tres experimentos donde bebés de distintas edades eran expuestos a sonidos de palabras en su idioma y sonidos de palabras en otro idioma, luego se medía el tiempo en el que el niño prestaba más atención y se observó que se quedaba fijo más tiempo en el idioma extranjero, reconociendo así su diferencia (los datos que encontraron los investigadores fueron una diferencia de ANOVA $F(1,34)=15.71 ; P<0.0005)$. Los resultados indican que a los 18 meses los bebés reconocen perfectamente su idioma, sobre todo en los sonidos consonánticos propios, en contraste con los sonidos consonánticos de otros idiomas.

En línea con el principio (v) Pascual, Aguado, Sotillo \& Masdeu (2008) evaluaron en un estu- dio longitudinal de dos años a 13 niños y 12 niñas mientras adquirían el español como lengua materna. En las evaluaciones que eran cada seis meses se le pedía a los padres de los niños que interactuaran con ellos en juegos de mesa y se grabó todo lo que los niños y los padres decían. Los investigadores encontraron que los padres modificaban sus expresiones habituales al hablar con los niños, lo que les ayudaba a adquirir palabras que les permitían indicar estados mentales propios o aj enos.

Pero además parece que los padres no solo ayudan al desarrollo del lenguaje, sino que evalúan apropiadamente el desarrollo de los niños. Esta afirmación se deriva del estudio de Mariscal, Nieva \& López-Ornat (2010) quienes al evaluar a 35 niños con el Mac-Arthur Bates y comparar los resultados arrojados por la prueba con un protocolo de observación que llenaban los padres, encontraron una alta correspondencia entre el instrumento de medición y el de observación, lo cual, para los investigadores implica una validez concurrente de la prueba, pero también se puede interpretar como una aguda observación y un particular interés de los padres por el desarrollo de los niños.

En línea con el principio antes expuesto (iii) Saffran, Senghas \& Trueswell (2001) afirman que desde los primeros periodos del desarrollo los bebés deben aprender a usar la materia prima del lenguaje que son los sonidos (o gestos) de su idioma, aprender cómo se ensamblan en cadenas más largas y el mapa de estas combinaciones en el significado. Estos procesos se desarrollan de forma simultánea, de modo que los niños integran sus capacidades a medida que aprenden, para descifrar el código de comunicación que les rodea.

Por último, y con relación al principio (iv), hay dos investigaciones que enfatizan en el acto de señalar como un hito muy importante en la vida de los bebés y en el posterior desarrollo del lenguaje. La primera es de Farkas (2007), quien afirma que los gestos refuerzan en vez de obstaculizar el desarrollo del lenguaje. El desarrollo de los gestos, tanto deícticos como simbólicos, se dan en un momento en que en el lactante aparece 
una necesidad de comunicarse, acompañada de un gran desarrollo cognitivo, pero en el cual la madurez fono respiratoria todavía no permite el surgimiento del habla. Así, los gestos cumplen un importante rol en esta etapa, precediendo, facilitando y complementando el desarrollo de los hitos del lenguaje.

La segunda es la realizada por Colonnesi, Stams, Koster \& Noom (2010), quienes en un meta-análisis sobre el tema seleccionaron investigaciones producidas entre 1978 y 2009, encontrando 25 estudios con una participación de 734 niños en total. Como resultado los investigadores afirman que hay una fuerte relación entre el gesto que aparece muy temprano en la infancia y el desarrollo del lenguaje. Lo anterior se debe a que el señalar se constituye como un acto de atención conjunta, siendo la primera forma de comunicación referencial e intencional.

En esta línea, los resultados de 25 estudios llevados a cabo en los últimos 30 años han demostrado una relación simultánea, así como una relación longitudinal entre señalar y lenguaje. Los resultados concurrentes proporcionan evidencia de que el gesto de señalar (que aparece fuertemente alrededor de los 11 meses) es una forma de comunicación que integra y apoya la adquisición del idioma. La relación longitudinal indica que los niños cuando señalan llaman la atención de las personas que les rodean y, a su vez, ellas les responden con palabras que el niño irá adquiriendo. De modo que el gesto de señalar es la primera forma de comunicación no lingüística y, al mismo tiempo, un comportamiento que ayuda a otras personas para comprender mejor y estimular el lenguaje de los bebés, en tanto, los introducen en un sistema comunicativo que les exige acoplarse en contextos relacionales psicológicos, culturales y afectivos (Peralta, 2000).

Pasando del estudio con bebés al estudio con niños un poco mayores, en la primera década del siglo XXI hay investigaciones que orientan sobre cómo continúa el desarrollo del lenguaje. El primero es el de Alonso (2000) quien al investigar los cambios en el lenguaje en tres grupos de niños de 3 a 6 años desde el modelo experimental de Luria, llega a la conclusión que los niños más pequeños acompañan su acción con expresiones en voz alta que les permiten guiar lo que hacen, mientras que los niños más grandes ya han interiorizado el lenguaje y esto les permite operar, hablando menos, pero con ayuda ocasional de producciones verbales; resultando congruente con lo que dice Gomila (2002) quien apoyado en Vigotsky, afirma que el lenguaje es un motor del desarrollo cognitivo en tanto apoya a la función ejecutiva y a la planeación de la acción.

Además de la posibilidad de planear la acción, Acuña, Nusser, \& Sentis (2009) afirman que hay otro hito en el desarrollo del lenguaje en etapas posteriores (niños en edad pre-escolar): el desarrollo de la referencia, entendida como la capacidad de remitirse 0 aludir a situaciones u objetos pasados, presentes y futuros. Este desarrollo de la referencia es fundamental para el dominio concreto de la dimensión semántica del lenguaje guiada por un proceso referencial. Es decir, gracias a la posibilidad de remitirnos a situaciones y objetos no presentes, se facilita la comprensión de los significados de las palabras que no necesariamente se remiten a obj etos del mundo, impulsando la dimensión semántica con cierta independencia de los objetos concretos.

Veamos entonces en que el lenguaje posibilita el control de la acción y que gracias a la capacidad referencial puede haber un adecuado desarrollo semántico. Ahora, hay un tercer elemento importante que viene dado con el desarrollo del lenguaje: la expresión y la materialización de la Teoría de la mente, entendida como la atribución de creencias, intenciones y deseos a otros individuos. Manzanares, Carbonero-Martín y Valle (2009) en una investigación realizada con 42 niños con una edad promedio de 4.8 años encontraron, después de realizar un análisis de tarea en problemas que implicaban teoría de la mente, que los niños con mejores capacidades lingüísticas resolvían mucho más fácil este tipo de problemas, hipotetizando que el lenguaje sirve de vehículo para la comprensión y solución de tareas mentalistas. Lo anterior se debe a que los signos usados por el lenguaje, materializan los contenidos mentalistas (intenciones y deseos) agilizando la 
comprensión y construcción de soluciones a los problemas. Según Crespo y Alfaro (2009) esta asociación entre teoría de la mente y lenguaje aparece en el desarrollo alrededor de los 506 años, cuando hay un gran paso en la socialización de los niños por su inclusión en entornos escolares más complejos.

La siguiente figura resume los principales aspectos expuestos hasta este momento en relación con la ontogénesis del lenguaje (figura 1):

Figura 1. Aspectos relevantes del desarrollo del lenguaje

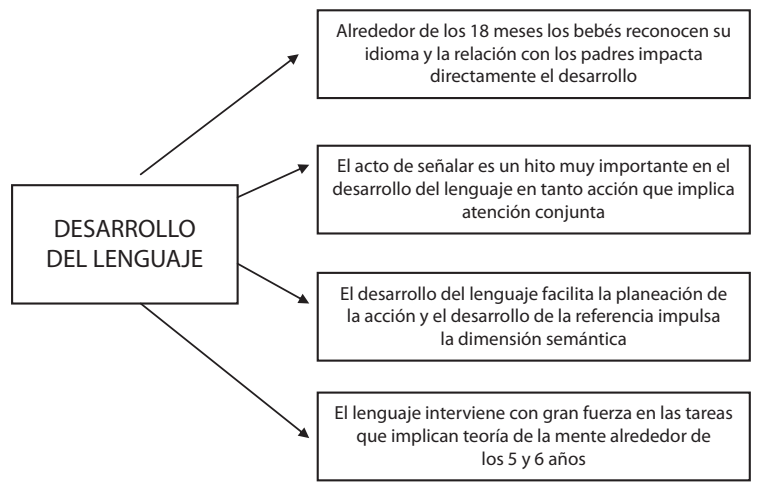

Estrechamente relacionado con el desarrollo del lenguaje en los niños; hay una serie de investigaciones que se han ocupado de estudiar el contexto social y familiar en el que el niño se desenvuelve, como un factor decisivo en el desarrollo. Dicho de otra manera, si bien es cierto que hemos aprendido más de las características del lenguaje en las primeras etapas de la ontogenia, no se puede perder de vista la importancia de un ambiente familiar y social benéfico para el niño.

Es así que cobra importancia investigaciones como la de Moreno (2005) quien se propuso dar cuenta de los efectos del maltrato infantil sobre el desarrollo del lenguaje, encontrando en una muestra de 39 menores con diferentes tipos de maltrato, que existen dificultades lingüísticas en los niños que lo han vivido, y dificultades que varían según la tipología del mismo, aunque el componente oral se encuentra afectado en todas.
En este orden de ideas, los menores en situación de maltrato físico presentan ligeras dificultades en morfología y los pertenecientes a la muestra de abandono físico en morfología y sintaxis. En cambio, los niños en situación de abandono emocional manifiestan considerables problemas en sintaxis, morfología y pragmática, al igual que en la muestra de maltrato emocional (Moreno \& García-Baamonde, 2009; Moreno, 2003).

En este punto cabe la pregunta ¿Por qué el maltrato infantil obstaculiza el desarrollo del lenguaje? Lo primero que puede decirse es que es debido a los problemas socioemocionales que induce, como por ejemplo: la agresión, la apatía y la frustración, hay una alteración general en el desarrollo (García, Rodríguez, Iglesias y Lodeiro, 2000). Pero otros estudios que indagan por la relación con los cuidadores y otros actores sociales pueden dar cuenta también del asunto. Por ejemplo Harty, Alant \& Uys (2006) encontraron que existe una correlación positiva entre la percepción materna de auto eficacia en la crianza y el desarrollo adecuado del lenguaje, llegando a la hipótesis que las madres que se perciben como más eficaces presentan menos frustración y tienen un abanico más amplio de recursos para la estimulación del lenguaje en los niños.

Lo anterior es compartido por Mcquaid, Bigelow, McLaughlin \& MacLean (2007) quienes estudiando el apego entre madres y niños concluyeron que las madres con apego seguro tienden con su lenguaje a promover la comprensión de estados mentales por parte de los niños, mientras que las madres con apego inseguro se les dificulta hacerlo. Esta apreciación es similar a la realizada por Ibañez (2003) quien al estudiar las posibles diferencias en el desarrollo del lenguaje entre niños aborígenes Mapuche y niños nacidos en Santiago de Chile, no encontraron diferencias significativas entre ambos grupos, pero afirman que es determinante la relación de apego establecida con la madre y la construcción del mundo por parte del niño de forma independiente al entorno cultural.

Ahora, no solo la relación directa con la madre afecta el desarrollo del lenguaje, parece incluso que la edad de gestación de la madre constitu- 
ye un factor decisivo. El estudio realizado por Keown, Woodward \& Field (2001) en el que comparaban el desarrollo de los niños hijos de madres adolescentes (menores de 20 años) y el desarrollo de los niños hijos de madres controles (entre 24 y 39 años) sostiene entre sus resultados que los niños nacidos de madres adolescentes obtuvieron puntuaciones significativamente más bajas en comprensión y expresión del lenguaje. Además en los antecedentes sociales, familiares se encontró que las madres adolescentes tenían una tasa más alta de embarazos no planificados, ingresos familiares menores y menor nivel de logros educativos en comparación con el otro grupo de madres. De igual forma, también se observó que las madres adolescentes proveían menos estimulación verbal a sus hijos y tenían un comportamiento menos sensible y más intrusivo, además de proporcionar a sus hijos un ambiente familiar menos estimulante, caracterizado por la disminución de la capacidad de respuesta emocional y verbal hacia estos.

Pero no solo la relación con la madre o los cuidadores primarios es importante para el desarrollo del lenguaje. La relación con los pares inducida en gran medida por la escolarización también juega un papel fundamental (Baquero 2009; Manrique y Rosemberg 2009). Lo anterior es apoyado por estudios como el de Galicia, Sánchez y Pavón (2009) quienes al evaluar el nivel de desarrollo psicolingüístico en 90 niños de los grados preescolar, primero y tercero de básica primaria; encontraron que a menor grado escolar, los niños presentaban un desarrollo psicolingüístico inferior a lo esperado para su edad, de modo que al parecer, la socialización con pares permite que haya un incremento en el desarrollo para ir alcanzado poco a poco los niveles esperados. Este énfasis en la importancia de la socialización viene dado por la estimulación y regulación que hacen los mismos niños de las emisiones lingüísticas, resultando que es beneficioso la inclusión de los niños en el sistema escolar (Mashburn, J ustice, Downer \& Pianta 2009; Campo 2010) y más si este contiene programas de estimulación del mismo (Herrera, Borges, Guevara \& Román, 2008; Sevcik, 2008; Rabazo \& Moreno, 2006).
En otra investigación hecha en Barranquilla Colombia, Campo (2009) encuentra una distribución muy irregular de habilidades lingüísticas en niños que ingresan al preescolar. Lo anterior se deduce después de evaluar a 229 niños entre 4 y 7 años asistentes a los grados: jardin, transición y primero entre 2007 y 2009 . Los datos de esta investigación afirman que:

En cuanto al lenguaje receptivo, el $65,3 \%$ de la muestra respondió de manera satisfactoria, presentando puntajes acordes a lo esperado para su edad. Sin embargo, se encontraron dificultades en el $24,3 \%$ de la muestra, que presentó un desarrollo por debajo de lo esperado, mientras que un $10,4 \%$ se ubicó por encima del parámetro. Por otro lado, en términos del lenguaje expresivo, que comprende la exploración de la articulación de los sonidos, lenguaje reflejado, la función nominativa del habla y el habla narrativa, el $76,7 \%$ de la muestra respondió de manera satisfactoria, pero se encontró una ligera dificultad en cuanto a la ejecución en esta área en el 18,4\% de la muestra, mientras que un $4,9 \%$ superó el grado de desarrollo esperado" (Campo, 2009, p. 345).

Esta irregularidad en la edad preescolar contrasta con la regularidad que se va presentando en niveles posteriores del desarrollo, lo que apoya la idea de lo beneficioso que resulta la inclusión escolar.

Ahora, es muy claro que tanto la relación madre hijo, como la vida escolar, están enmarcadas en características sociales y económicas más amplias que también inciden en el desarrollo del lenguaje. El estudio de Pérez (2004) en el que tomó 92 niños de 4-5 años pertenecientes a los estratos 1, 2, 3 y 4 para ser observados en su institución educativa, propone que los niños pertenecientes a estratos socio-económicos 1-2 muestran un desfase en los niveles del lenguaje en comparación con los niños de la misma edad de estratos 3-4, debido a que presentan más omisiones, sustituciones, transposiciones y contaminaciones en su desempeño lingüístico. 
No se puede dejar de lado, en el análisis que se viene haciendo, una variable muy importante que además resulta predictiva en cuanto el desarrollo del lenguaje: la nutrición. En el meta-análisis realizado por Barre, Morgan, Doyle \& Anderson (2010), que comprendía artículos entre 1990 y 2009 en los que se buscaba establecer diferencias entre el posterior desarrollo del lenguaje de niños nacidos prematuros (menos de 32 semanas de gestación), niños con un peso muy bajo al nacer (menor a 1.500 gramos) y niños controles nacidos a término y sin problemas nutricionales. Los resultados del meta-análisis permitieron afirmar que los niños prematuros y con bajo peso tienden a tener menores puntuaciones en lenguaje expresivo que los grupos controles. Aspecto que se repite en el lenguaje receptivo y el posterior desempeño escolar. Sin embargo, los autores afirman que una rápida intervención que permita ganar peso a los niños y normalizar las características de haber nacido prematuros, disminuye el riesgo de problemas del lenguaje en el fututo.

Resumiendo, es pertinente afirmar que condiciones nutricionales, familiares, escolares y sociales favorables repercuten positivamente en el desarrollo del lenguaje y este a su vez contribuye positivamente en otros aspectos de la vida. Al respecto, el trabajo realizado por Hohm, J ennen, Schmidt \& Laucht (2007), quienes en un gran estudio longitudinal de más de 10 años en el que evaluaron a 90 niños desde que tenían 9, 3 meses hasta que tenían 11 años, encontraron, siguiendo el análisis de regresión múltiple que los niños que mejor puntuaron en el desarrollo del lenguaje obtuvieron luego en la escuela mejores desempeños en matemáticas, en aprendizaje del alemán y en ortografía. Por lo cual se puede afirmar que un mejor desarrollo del lenguaje es predictivo de un mejor desempeño en dichas áreas escolares.

Ahora, decir que un mejor desarrollo del lenguaje está relacionado con otras características del niño, lleva a plantear la pregunta ¿cuáles son las diferencias existentes entre los niños con un desarrollo normal del lenguaje, respecto a un desarrollo anormal? La primera investigación que habla del tema es la de Flórez, Mesa y Torrado (2006), en la que compararon capacidades me- talingüísticas entendidas como la posibilidad de analizar críticamente el contenido o el uso del lenguaje en particulares contextos comunicativos. Participaron en el estudio niños típicos, institucionalizados y con deficiencia intelectual, encontrando que estos últimos tienen una marcada alteración en todos los indicadores de capacidades metalinguisticas.

Además de lo anterior, los niños con un desarroIlo anormal del lenguaje, presentan también más dificultades en situaciones que implican atención conjunta y expresión de intenciones (Topba, Mavi \& Erba, 2003), más dificultades en la relación con sus madres (Melgarejo, Molina y López, 2005; Henao y García, 2009; Vite, López y Negrete, 2010) y mayores problemas en la socialización con los pares de modo que suelen confiar más en los adultos que en otros niños para la interacción (McCabe \& Marshall, 2006).

En otro estudio, Van Dall, Verhoeven \& Van Balkom (2007) tomaron al azar 97 niños con problemas del lenguaje procedentes de escuelas de educación especial de los países bajos, sin tener en cuenta para el estudio a niños con problemas motores y con trastornos generalizados del desarrollo, encontraron que los problemas de sintaxis correlacionaron positivamente con problemas de pensamiento y atención; mientras que problemas en la semántica tienen correlaciones significativas con aislamiento social, ansiedad y depresión. Finalmente, los problemas fonológicos mostraron correlación con problemas en comportamientos sociales como conducta delictiva y conducta agresiva.

\section{Conclusiones}

Después de retomar las investigaciones publicadas entre 2000 y 2010, puede concluirse en primer lugar que hay mayor claridad respecto al origen social del lenguaje, constituyéndose así en un proceso que apoya la adaptación a los grupos y que requiere de entornos sociales adecuados para su desarrollo en el individuo. 
Pero la dimensión social no es suficiente para entender la aparición y desarrollo del lenguaje, se necesitan de estructuras biológicas que apoyen este proceso. En ese sentido puede afirmarse (con mayor certeza que en otros momentos históricos) que en términos anatómicos el lenguaje requiere de la participación de los lóbulos temporal y frontal.

Teniendo en cuenta esta combinación de aspectos sociales y anatómicos, diversas investigaciones confluyen en sostener que en términos del desarrollo ontogenético del lenguaje se conocen los siguientes hitos importantes: (A) los bebés participan activamente en el reconocimiento de su lengua lográndolo alrededor de los 18 meses. (B) El acto de señalar impulsa la atención conjunta y el intercambio lingüístico. (C) Los niños se valen del lenguaje para resolver tareas de teoría de la mente y aproximadamente a los 5-6 años empiezan a reconocer actos de habla indirectos e ironías.

Finalmente, puede concluirse que el desarrollo del lenguaje está influido por variables como la nutrición, la relación con los padres, el ambiente escolar y los entornos sociales más macro, afectándolo positiva o negativamente, dependiendo de la expresión de estas en las primeras etapas de vida.

\section{Referencias}

Acuña, X., Nusser, C. y Sentis, F. (2009). El desarrollo del lenguaje y el desarrollo de la referencia en la adquisición de la lengua materna. Onomazein, 2(20), 147-191.

Alonso, L. (2000). El papel del lenguaje interior en la regulación del comportamiento. Educere, 3(009), 61-68.

Baquero, R. (2009). Desarrollo psicológico y escolarización en los enfoques socioculturales: nuevos sentidos de un viejo problema. Avances en Psicología Latinoamericana, 27(2), 263-280.
Barre, N., Morgan, A., Doyle, L. \& Anderson, P. (2010). Language Abilities in Children Who Were Very Preterm and/or Very Low Birth Weight: A Meta-Analysis. J ournal of pediatrics, 158(5), 766-774.

Campo Ternera, L. (2009). Características del desarrollo cognitivo y del lenguaje en niños de edad preescolar. Psicogente, 12(22), 341-351.

Campo Ternera, L. (2010). Relación entre el desarrollo personal social y los procesos evolutivos vinculados con el aprendizaje escolar en las áreas del lenguaje y la cognición. Psicogente, 13(23), 88-99.

Castaño, J. (2003). Bases neurobiológicas del lenguaje y sus alteraciones. Revista de Neurología, 36(8), 781-787.

Colonnesi, C., Stams, J., Koster, I. \& Noom, M. (2010). The relation between pointing and language development: A meta-analysis. Developmental Review, 30(4), 352-366.

Crespo Allende, N. \& Alfaro, P. (2009). La conciencia meta pragmática en la edad escolar. Boletín de Lingüística, 21(32), 7-24.

Dehaene, S., Artiges, E. \& Naccache, L. (2003). Conscious and subliminal conflicts in normal subjects and patients with schizophrenia: the role of the anterior cingulate. Proceedings of the National Academy of Sciences of the United States of America - PNAS, 100(23), 13722-13727.

De Vega, M. (2005). Lenguaje, corporeidad y cerebro: una revisión crítica. Revista Signos: Estudios de Lingüística, 58, 157-176.

Fajardo, L. (2008). Aproximación a la relación entre cerebro y lenguaje. Cuadernos de Lingüística Hispánica, 11, 93-104.

Farkas, C. (2007). Comunicación gestual en la infancia temprana: una revisión de su desarrollo, relación con el lenguaje e implicancias de su intervención. Psykhe, 16(2), 107-115. 
Flórez, R., Mesa, C. y Torrado, M. (2006). Emergencia de las capacidades metalingüísticas. Revista Latinoamericana de Psicología, 38(3), 457-475.

Galicia Moneda, I., Sánchez Velasco, A. y Pavón Figueroa, S. (2009). Habilidades psicolingüísticas al ingreso y egreso del jardín de niños. Revista Intercontinental de Psicología y Educación, 11(2), 13-36.

García, C., Rodríguez, P., Iglesias, M.J . y Lodeiro, O. (2000). Problemas socioemocionales y habilidades lingüísticas en preescolares. Psicothema, 12(3), 412-417.

Goldin-Meadow, S., Chee So, W., Zyurek, A. \& Mylander, C. (2008). The natural order of events: How speakers of different languages represent events nonverbally. Proceedings of the National Academy of Sciences of the United States of America - PNAS, 105(27), 9163-9168.

Gomila Benejan, A. (2002). El lenguaje del pensamiento conciente. Querk: Ciencia, medicina, comunicación y cultura, 25, 26-38.

Harty, M., Alant, E \& Uys C. (2006). Maternal selfefficacy and maternal perception of child language competence in pre-school children with a communication disability. Child: Care, Health and Development, 33(2), 144-154.

Henao, G., García, M. (2009). Interacción familiar y desarrollo emocional en niños y niñas. Revista Latinoamericana de Ciencias Sociales, Niñez y J uventud, 7(2), 785-802.

Herrera Rodríguez, J., Borges Rodríguez, S., Guevara Fernández, G. \& Román Cao, I. (2008). La estimulación del desarrollo del lenguaje en la edad preescolar, una propuesta desde su componente léxico-semántico. Revista Iberoamericana de Educación, 47, 1-12.

Hohm, E., J ennen Steinmetz, C., Schmidt, M. \& Laucht, M. (2007). Language development at ten months: Predictive of language outcome and school achievement ten years later. European Child \& Adolescent Psychiatry, 16(3), 149-156.

Ibañez, N. (2003). La construcción del mundo en el lenguaje. Revista de Psicología. Universidad de Chile, 12(002), 71-84.

Junyet, C. (2001). Evolución, lenguaje y conocimiento. Quark Ciencia, medicina, comunicación y cultura, 21, 17-19.

Keowm, L. J., Woodward, L. \& Field, J. (2001). Language development of pre-school children born to teenage mothers. Infant and Child Development, 10, 129-145.

Kinzler, K., Dupoux, E. \& Spelke, E. (2007). The native language of social cognition. Proceedings of the National Academy of Sciences of the United States of America - PNAS, 104(30), 12577-12580.

Kirby, S., Hannah, C. \& Smith, K. (2008). Cumulative cultural evolution in the laboratory: An experimental approach to the origins of structure in human language. Proceedings of the National Academy of Sciences of the United States of America - PNAS, 105(31), 10681-10686.

Kuhl, P. (2000). A new view of language acquisition. Proceedings of the National Academy of Sciences of the United States of America - PNAS, 97(22), 11850-11857.

Manrique, M., Rosemberg, C. (2009). El lenguaje infantil en situaciones de juego en el J ardín de Infantes. Summa Psicológica, 6(2), 105-118.

Manzanares, M, C., Carbonero-Martín, M. \& Valle, L. (2009). Análisis del procesamiento en tareas tradicionalmente cognitivas y de teoría de la mente en niños de 4 y 5 años. Psicothema, 22(4), 772-777.

Mariscal Altares, S., Nieva, S. \& López-Ornat, S. (2010). Observar y medir el desarrollo gra- 
matical temprano en español. Psicothema, 22(1), 51-56.

Mashburn, A., J ustice, L., Downer, J. \& Pianta, R. (2009). Peer effects on children's language achievement during pre-kindergarten. Child Development, 80(3), 686-702.

McCabe, P. \& Marshall, D. (2006). Measuring the social competence of preschool children with specific language impairment. Topics in Early Childhood Special Education, 26(4), 234-246.

Mcquaid, N., Bigelow, A., McLaughlin, J. \& MacLean, K. (2007). Maternal mental state language and preschool children's attachment security: Relation to children's mental state language and expressions of emotional understanding. Social Development, 17(1), $1-24$.

Melgarejo, C., Molina, C. \& López, F. (2008). Interacción madre-hijo: una comparación de niños con y sin problemas de lenguaje. Enseñanza e Investigación en Psicología, 10(01), 21-38.

Montealegre, S. (2005). La actividad humana en la psicología histórico-cultural. Avances en Psicología Latinoamericana, 23, 33-42.

Moreno Manso, J. (2003). Estudio sobre las repercusiones lingüísticas del maltrato y abandono emocional infantil. Revista de Logopedia, Foniatría y Audiología, 23(4), 211-222.

Moreno Manso, J. (2005). Estudio sobre las consecuencias del maltrato infantil en el desarroIlo del lenguaje. Anales de Psicología, 21(2), 224-230.

Moreno, J.M. \& García-Baamonde, M.E. (2009). Adaptación personal y desarrollo lingüístico en niños víctimas de maltrato. Boletín de Psicología, 96, 17-34.

Pascual, B., Aguado, G., Sotillo, M. \& Masdeu, J. (2008). Acquisition of mental state language in Spanish children: a longitudinal study of the relationship between the production of mental verbs and linguistic development. Developmental Science, 11(4), 454-466

Peña-Garay, M. (2005). Habilidades lingüísticas de los niños menores de un año. Revista de Neurología, 40, 1-8.

Peralta, J. (2000). Adquisición y desarrollo del lenguaje: una visión pragmática constructivista centrada en los contextos. Límite, 007, 54-66.

Pérez, M. (2004). Influencia de factores ambientales en el desarrollo del lenguaje. Umbral Científico, 005, 43-49.

Perruchet, P. (2005). Statistical approaches to language acquisition and the self-organizing. Psychological Research, 69, 316-329.

Pettito, L., Holowka, S., Sergio, L. (2001) Language rhythms in baby hand movements. Nature, 413(6851), 35-38.

Pinker, S. (2010). The cognitive niche: Coevolution of intelligence, sociality, and language. Proceedings of the National Academy of Sciences of the United States of America PNAS, 107(2), 8993-8999.

Rabazo, M., Moreno, J. (2006). Intervención psicopedagógica en niños maltratados. Estimulación del lenguaje. Electronic J ournal of Research in Educational Psychology, 4(8), 83-105.

Saffran, J., Senghas, A. \& Trueswell, J. (2001). The acquisition of language by children. Proceedings of the National Academy of Sciences of the United States of America PNAS, 98(23), 12874-12875.

Scerif, G. (2006) ¿Qué tienen de especial los módulos? Hacia una definición de los mecanismos de cambio en el desarrollo. Infancia y Aprendizaje, 29(3), 281-288. 
Sevcik, R. A. (2008). Comprehension: An overlooked component in augmented language development. Disability and Rehabilitation, 28(3), 159-167.

Spinney, L. (2003). Investigan vínculo evolutivo entre el habla y el lenguaje del cuerpo. Educere, 6(20), 441-442.

Sullivan, J., Riccio, C. (2008). Language functioning and deficits following pediatric traumatic brain injury. Applied Neuropsychology, 17(2), 93-98.

Topba, S., Mavi. I., Erba. D. (2003). Intentional communicative behaviours of Turkishspeaking children with normal and delayed language development. Child: Care, Health \& Development, 29(5), 345-355.
Van Daal, J., Verhoeven, L. \& Van Balkom, H. (2007). Behaviour problems in children with language impairment. Journal of Child Psychology and Psychiatry, 48(11),1139-1147.

Vigneau, M., Beaucousin, A., Hervea, P. \& Duffauc, H. (2006). Meta-analyzing left hemisphere language areas: Phonology, semantics, and sentence processing. Neuroimage, 30, 1414-1432.

Vite, A., López, F. y Negrete, A. (2010). Sensibilidad materna y maltrato infantil. Acta Colombiana de Psicología, 13(2), 11-18.

Waibel, A. (2001). Los sistemas integrales completos del habla, del lenguaje y la interfaz humana. Quark Ciencia, medicina, comunicación y cultura, 21, 95-102. 